\title{
Factores vocacionales que influyeron en estudiantes mujeres de la UNA al escoger la Ingeniería en Topografía y Catastro
}

\section{Vocational factors that influenced female students of the UNA when choosing Engineering in Topography and Cadastre}

\author{
José Pablo Calero-Torres \\ Instituto Nacional de Aprendizaje \\ San José, Costa Rica \\ josepablo1596@hotmail.com \\ https://orcid.org/0000-0001-8202-9496
}

RECIBIDO: 9 de julio de 2021 / ACEPTADO: 6 de noviembre de 2021

\section{Resumen}

La sociedad actual ha realizado múltiples cambios durante esta década al romper barreras ideológicas y algunos estereotipos. Uno de estos es la incursión de mujeres en carreras no tradicionales para su sexo como es el caso de las ingenierías. Por esta razón, surge el interés de realizar esta investigación para determinar la influencia de los factores vocacionales en la elección profesional de estudiantes mujeres de primer nivel de la carrera de Ingeniería en Topografía y Catastro de la Universidad Nacional; y asimismo, para reconocer el motivo de su elección. La investigación se realizó mediante un paradigma naturalista y un enfoque cualitativo por las características que poseen. De esta manera se evidencia, entre los hallazgos, que el factor que menos influencia posee en la elección vocacional es el socioeconómico: la familia. Aunque es un elemento importante por la dinámica interna, no ejerce la presión necesaria para intervenir en la decisión de las estudiantes, ya que aún existen estereotipos en la sociedad hacia las mujeres. Con este trabajo se pretende crear conciencia en las personas lectoras sobre la capacidad del cambio; y evidenciar la incorporación de las mujeres en espacios con predominancia de hombres en el ámbito académico y ámbito laboral como un fenómeno actual.

Palabras clave: orientación vocacional, factores vocacionales, elección de carrera, género.

\section{Abstract}

Today's society has made multiple changes during the current years, breaking ideological barriers and some stereotypes, within which there is the incursion of women into non-traditional careers for their sex, such as engineering. It is for this reason that the interest arises to conduct this research. With the general purpose of determining the influence of vocational factors in the professional choice of first-level female students of the Engineering in Topography and Cadastre at the National University, to recognize the reason for their choice. This research was carried out using a naturalistic paradigm and a qualitative approach due to the characteristics they possess, among the findings identified it is mentioned that the factor that least influences the vocational choice was the socioeconomic one, the family. Although it is an important element due to the internal dynamics, it does not exert the necessary pressure to intervene in the decision of the students and, nonetheless, there are still stereotypes in society towards women. This work aims to create awareness in readers about the capacity for change. To show a current phenomenon, which is the incorporation of women in spaces with a predominance of men in the academic field and, later, in the workplace.

Keywords: Vocational guidance; vocational factors; career choice; gender. 


\section{Revista Costarricense de Orientación ISSN 2215-6615 • Vol. 1 Num. 1, Enero-Junio, 2022, p. 1-15 \\ Calero-Torres \\ https://doi.org/10.54413/rco.v1i1.16}

\section{Introducción}

Históricamente han existido algunas carreras universitarias en las cuales existe mayor predominancia de un sexo que otro. Esta situación ha creado estereotipos o estigmas, tal como se indica en el estudio realizado en México por el Instituto Nacional de Estadística e Informática (2012):

$\mathrm{Al}$ analizar según sexo, las carreras universitarias o profesiones de mayor preferencia por las mujeres son las siguientes: Educación 27,4\%, mayor en 13,8 puntos porcentuales al de los hombres; Ciencias Contables y Financieras con 10,8\%, a diferencia de los hombres que llega a 10,2\%. La carrera de Enfermería en 6,8\% mayor en 6,0 puntos porcentuales al de los hombres (0,8\%), Psicología 3,3\% en las mujeres y $1,7 \%$ en los hombres. Asimismo, la carrera de Obstetricia es escogida por las mujeres, en $2,9 \%$, siendo mayor en 2,7 puntos porcentuales al porcentaje observado en los hombres. (p. 122)

El estudio anterior evidencia y respalda que algunas profesiones son acaparadas en su mayoría por mujeres; y que además, esta situación no ocurre únicamente a nivel nacional sino a también a nivel internacional. Otro dato revelador del estudio se basa en los tipos de profesiones en las cuales se gradúan las jóvenes, como: áreas de salud, educación y numéricas. En comparación a sus colegas hombres el mismo estudio indica que:

En los hombres, las carreras de mayor preferencia que escogieron para estudiar o han estudiado son: Ingeniería 28,3\%, a diferencia de las mujeres que representa el 7,6\%; Administración de Empresas $10,9 \%$ en los hombres y $8,5 \%$ en las mujeres, siendo 2,4 puntos porcentuales menor que de los hombres; Derecho y Ciencias Políticas con $8,7 \%$ en los hombres y $8,2 \%$ en las mujeres. Asimismo, hay 3,6\% de hombres que estudian o han estudiado Economía, mientras que en las mujeres lo hicieron el 1,9\%; Medicina Humana y otras carreras relacionadas a la medicina representan el 4,5\% de los hombres y el 2,3\% de las mujeres, Medicina Veterinaria y Zootécnica (3,5\%), Arquitectura y Urbanismo $(1,6 \%)$ son carreras con mayor preferencia por los hombres que las mujeres, entre las principales. (p. 123)

En esta contraposición se evidencia la preferencia de parte de los hombres por carreras en áreas de ingenierías, administración e incluso en áreas de ciencias políticas a diferencia de los porcentajes en la inscripción de las mujeres. Así, se observa que estas carreras son masculinizadas por la predominancia masculina.

Al existir una mayor cantidad de un sexo en una carrera universitaria surgen estereotipos o creencias sobres las mismas; como, por ejemplo, que las carreras y profesiones relacionadas a la educación le corresponde únicamente a las mujeres o que las ingenierías, debido a la fuerza física para transportar algún material, es solo para los hombres. Por eso, cuando una persona ingresa a una carrera en donde predomina el sexo opuesto se rompe este paradigma, pero recae sobre ella el señalamiento.

Como es planteado por Cubillas, Valdez, Domínguez, Román, Hernández y Zapata (2016): "los estereotipos de género son un conjunto estructurado de creencias compartidas dentro de una cultura o grupo acerca de los atributos o características que posee cada sexo" (p. 218). Es importante enfatizar que estos estereotipos, como lo mencionan las personas autoras, son constructos creados por un grupo de la sociedad dirigido hacia otro grupo. Estos persisten a través del tiempo, son constantes, acogidos como realidad; por ejemplo, se puede mencionar que las mujeres son débiles, sin embargo, la problemática surge cuando se trasciende al sexismo, el cual para fines de la investigación se entenderá según los postulados de Mejías (2017) como: 
Es la discriminación que se realiza a un hombre o a una mujer solo por pertenecer a un género, es un prejuicio basado en el sexo masculino o femenino, todas estas actitudes están basadas en estereotipos de roles sociales.

El sexismo se originó en el entorno del feminismo en la década de los sesenta, para referirse a los patrones que se habían creado sobre la superioridad de un sexo frente al otro. (p. 18)

Con la intención de crear una compresión amplia sobre el término sexismo, se explica que el mismo surge cuando se denigra a un sexo específico a partir de creencias, estereotipos y estigmas de una persona o un colectivo. No obstante, evoluciona de discriminación al sexismo cuando se empieza a minimizar al sexo contrario e inician conductas discriminantes o despectivas directas hacia esa población única y específicamente por su sexo.

Por esta razón surge el interés de conocer el motivo de la matrícula de parte de mujeres en esta profesión, además del factor vocacional que intervino en esta elección no tradicional para su sexo. Por consiguiente, se plantea la siguiente pregunta:

- ¿Cómo inciden los factores vocacionales en la elección profesional de estudiantes mujeres de primer nivel de la carrera de Ingeniería en Topografía de la Universidad Nacional en el año 2020?

Con la finalidad de brindar una respuesta, se ejecuta el propósito general:

- Determinar la influencia de los factores vocacionales en la elección profesional de estudiantes mujeres de primer nivel de la carrera de Ingeniería en Topografía y Catastro de la Universidad Nacional para reconocer el motivo de su elección.

Y además, se establecen los propósitos específicos:

1. Indagar los factores personales que inciden en la elección de carrera de la población participante.

2. Identificar factores familiares que se relacionan con la elección de las estudiantes sobre la carrera.

3. Reconocer los factores sociales que inciden en la elección de esta carrera.

\section{Referencia teórica}

Para una comprensión clara se desarrolla el concepto de orientación vocacional. Se recopila a Galilea (s.f.), quien cita a los autores Anthony y Cols (1984), y los cuales plantean que:

La Orientación Vocacional es un proceso que tiene como objetivo despertar intereses vocacionales, ajustar dichos intereses a la competencia laboral del sujeto y a las necesidades del mercado de trabajo. El primer paso de la rehabilitación vocacional es la elección de un interés realista que permita al sujeto alcanzar su meta laboral. (p. 1)

La orientación vocacional es una rama de la disciplina de orientación, y contribuye a que la población orientada en el sistema educativo costarricense donde se encuentre este profesional culmine esta etapa de su vida con un amplio conocimiento en el área laboral, como: ofertas académicas, mercado laboral.

Asimismo, abarca aspectos propios como el autoconocimiento, capacidades, habilidades, aptitudes y actitudes, según corresponda su nivel académico de acuerdo con los programas de orientación, con la finalidad de que los estudiantes realicen una escogencia óptima en el mundo laboral. 
Dentro de este conocimiento entran aspectos como la opinión e influencia de familiares, las decisiones de los mismos y el ambiente. Por esta razón, se engloban tres grandes factores con sus propios elementos o unidades de análisis:

\section{Factor personal}

Es el único factor en el cual sus elementos se interrelacionan en la misma persona, y se encuentran a nivel intrínseco:

\section{Intereses}

Estos se entenderán de acuerdo con Reyes y Novoa (2014) como:

1. Frecuencia con la que alguien se comporta de manera usual o desde su cotidianidad. Por ejemplo, se dice que "a Pepe le interesa el fútbol" cuando observamos que juega fútbol dos horas diarias y que ve o escucha por lo menos el $70 \%$ de los partidos de sus equipos favoritos.

2. Grado de placer que se experimenta al comportarse de determinada manera en ciertas ocasiones. Por ejemplo, se dice que "a Sofía le interesa tocar el piano" cuando, independientemente de la frecuencia con que toque el piano, ella manifiesta que le gusta mucho hacerlo. (p. 14)

El interés se considera una fuerza que mueve a las personas, y surge de la atracción o el deseo de cada quien por alcanzar una meta, culminar o realizar un sueño. La realización del mismo representa en alguna medida un logro, como se considera anteriormente en los ejemplos que comentan los autores. Por lo tanto, la carrera en Ingeniería en Topografía y Catastro cautiva a la población participante, es de su interés y por dicha razón se inscribe en la misma.

\section{Autoconocimiento}

Este elemento es mucho más amplio en su esencia. Se considera a cada ser humano como un ser holístico, en el cual cada una de sus partes (alma, espíritu y cuerpo) se relacionan entre sí.

De aquí surge la importancia de conocerse, ya que este proceso brinda claridad en la toma de decisiones. López (2003, citado por Márquez, 2016) lo define como:

El autoconocimiento, que es la capacidad para tomar conciencia del estado emocional propio y de expresarlo con lenguaje verbal y no verbal. A través del autoconocimiento somos capaces de reconocer nuestras emociones y sentimientos y nos ayuda a reconocer las de los demás. (p. 5)

Cuando la persona logra desarrollar un autoconocimiento, claramente, se conoce a sí mismo; y conoce sus capacidades, habilidades, debilidades, fortalezas, amenazas. Además, logra reconocer oportunidades, valora situaciones como la capacidad de permanecer o no en la profesión, identifica si posee las habilidades numéricas solicitadas en los planes de estudio, entre otros.

\section{Factor familiar}

El segundo factor para considerar involucra a los miembros del núcleo familiar, estos elementos son extrínsecos de las jóvenes. 


\section{Influencia y comunicación familiar}

Estos elementos se desarrollaron por aparte en los instrumentos de recolección de información, sin embargo, para su desarrollo teórico se considera pertinente unificarlos.

La influencia familiar se investigó como la capacidad de los miembros en interferir sobre la elección vocacional de las participantes. De esta manera, se consideraron opiniones, consejos, presión ejercida por parte de las figuras de autoridad (sean estos actos con la intención de hacer cambiar el pensamiento de las jóvenes o hacerles elegir esta profesión).

Se valora la comunicación de acuerdo con la teoría realizada por Tustón (2016):

La comunicación es de suma importancia en el ámbito familiar, a través de la comunicación establecemos contacto con las personas, transmitimos nuestros pensamientos y sentimientos, es este sentido todos por muy distintos que seamos, queremos ser escuchados, comprendidos y tenidos en cuenta. (p. 1)

La comunicación tanto verbal como no verbal brinda un espacio de intercambio de información, permite expresarnos hacia un receptor, crea vínculos más sólidos al confiar en estas fuentes cierta información e incluso solicitar recomendaciones o consejos ante una duda y la incertidumbre de una escogencia de carrera universitaria en este caso, por lo tanto, este aspecto entra en juego en la investigación y se relaciona con la influencia familiar.

\section{Factor social}

El factor social está directamente relacionado con el ambiente en el cual se desenvuelven las participantes, entendiendo este como su lugar de procedencia, casa de habitación y cualquier otro elemento del ambiente físico en donde viva junto a sus características. Al igual que el anterior factor es extrínseco, los elementos presentados son externos pero considerados decisivos para la toma de la decisión ya que pueden influir por un tema modelaje con sus vecinos, esto con carácter de ejemplo.

\section{Conocimiento del medio}

Hace énfasis a la capacidad de las jóvenes de reconocer el ambiente donde se desenvuelven o aspiran hacerlo, se relaciona con la demanda laboral de profesión donde se van a desempeñar. En el presente se vincula con la carrera donde se encuentran, el ambiente educativo, sus oportunidades y debilidades relacionadas al estudio.

$\mathrm{Al}$ ser mujeres en un espacio no tradicional deben ser conscientes del espacio donde se encuentran y las repercusiones tanto positivas como negativas de esta elección y sus consecuencias a futuro, puesto que al poseer este conocimiento podrán desenvolverse con mayor agilidad.

\section{Medios socioeconómicos}

Álvarez (2017) afirma que:

En lo referente a la disponibilidad económica es realmente un factor preponderante que imposibilita al estudiante hacer una elección independiente de su carrera, esto ha hecho que muchos opten o se conformen por seguir estudios para los cuales no tienen interés, ni mucho menos aptitudes y por habilidades que permitan cumplir su meta profesional. (p. 12) 
En ocasiones un nivel económico bajo podría impedir realizar estudios de formación superiores por medio de un financiamiento propio incurriendo en préstamos o en algunos casos la obtención de una beca, en otros casos podría estimular la escogencia de una carrera con algún tipo de prestigio en la sociedad o con salarios altos. Es por esta razón que se consideró como parte del factor social, por su capacidad de influir en la elección de las estudiantes en la carrera en estudio.

\section{Metodología}

Después del sustento teórico que respalda la investigación se inicia el proceso metodológico de la recolección de los datos. Para esto se contactan a las cinco participantes para un muestreo de voluntarias.

Se prosigue con la coordinación para la aplicación de los instrumentos. Primeramente, se aplica la entrevista de acuerdo con el enfoque cualitativo. Esta se realiza de forma individual con cada una de las participantes por medio de una cita agendada, y el instrumento está compuesto por un total de 19 ítems que responden los tres factores en estudio, y los cuales se designan de la siguiente forma:

\section{Tabla 1}

Operación de los items

\begin{tabular}{lll}
\hline \multicolumn{1}{c}{ Categoría } & \multicolumn{1}{c}{ Sub categoría } & \multicolumn{1}{c}{ Ítem } \\
\hline \multirow{2}{*}{ Factores personales } & Intereses & $2,4,18$ \\
& Autoconocimiento & $1,3,5$ \\
& Influencia familiar & $11,13,15$ \\
Factores familiares & Comunicación familiar & $6,12,16$ \\
& Conocimiento del medio & $7,8,10$ \\
Factor social & Factores socioeconómicos & $14,17,9$ \\
\hline
\end{tabular}

El segundo instrumento fue una historia de vida, es uno de los instrumentos que permite mayor expresión, ya que en una serie de páginas (según la cantidad solicitada como máximas) redactarán, de forma clara, su vivencia sobre el proceso vocacional desde los tres factores en estudio. Al respecto Hernández, Fernández y Baptista (2014) consideran:

Muy socorrida en la investigación cualitativa. Puede ser individual (un participante o un personaje histórico) o colectiva (una familia, un grupo de personas que vivieron durante un periodo y que compartieron rasgos y vivencias). Para realizarla se suele utilizar entrevistas en profundidad y revisión de documentos y artefactos personales e históricos. (p. 416)

Como se ha mencionado, anteriormente, la investigación es de enfoque cualitativo, y es un eje primordial no confundirlo con el cuantitativo. La aplicación de ambos instrumentos se realizó de forma 
individual con cada una de las participantes; en cuanto a las historias de vida, se les entregaron los lineamientos en formato físico para ese instrumento el día que se realizó la entrevista. Asimismo, se otorgaron 15 días hábiles para la realización y entrega, y se informaron los medios para contactar al investigador en caso que surgiera dudas durante el proceso de deconstrucción del instrumento. Además, se les menciona sobre la confidencialidad de los datos por recolectar.

Considerando la extensión del instrumento y el tiempo requerido para su respectivo análisis, se plantean lineamientos claros para quelas estudiantes puedan brindar la información necesaria. La metodología de aplicación consiste en forma individual, y se les solicita que adjunten (si lo desean) documentos, fotos o algún dato relevante o pertinente a los factores y al tema abordado, ya que este debe estar estrictamente vinculado para así evitar información innecesaria que demore la entrega del instrumento.

Con la finalidad de comprender la estructura metodológica que posee la investigación se explica a continuación:

\section{Paradigma de investigación}

Para efectos metodológicos del trabajo, en donde se considera la recolección de los datos, la selección de los instrumentos y el análisis de los resultados, se ha seleccionado el paradigma naturalista; ya que este responde a los propósitos por realizar en esta investigación.

Sobre el paradigma Schuster, Puente, Andradra y Maiza (2013) consideran que:

El término paradigma hace referencia al conjunto de creencias y actitudes, como una visión del mundo compartida por un grupo de científicos. Cada comunidad de científicos comparte un mismo paradigma y conforma, de esta manera, una comunidad intelectual cuyos integrantes tienen en común valores, creencias, normas, objetivos, un lenguaje determinado; en definitiva, una metodología. (p. 119)

La selección de un paradigma se debe realizar con determinación, puesto que este marcará el camino de la investigación, además, posee elementos fundamentales como el enfoque y el diseño. Cada paradigma contiene una visión del procedimiento como es la recolección de datos que le corresponden.

Existen diversos tipos de paradigmas que se pueden seleccionar. Esto va a depender de la investigación, de la forma en la cual se recolectará la información y de cómo se quiere presentar. Por lo que se indaga sobre cada una de ellas, y finalmente se selecciona la que se considera pertinente por sus características, lo que permite una mayor cercanía con la muestra de la población y de la interacción que surgió durante el proceso.

Se desea conocer la realidad de las participantes, con frases etnográficas, involucrando sus propias formas de vivir el proceso de elección de la carrera, el proceso de admisión y el posterior ingreso a la universidad, por estos motivos se escoge el paradigma naturalista.

Fraile y Vizcarra (2009) plantean que: "La metodología naturalista ayuda a la comprensión e interpretación de una realidad educativa concreta, despreocupándose de las relaciones causa-efecto. El afán por comprender las realidades educativas cotidianas nos hace optar por estudios naturalistas y etnográficos” (p. 122).

Debido a que se desea conocer la influencia de los factores vocacionales en la elección de la población participante, se presta importancia al fenómeno desarrollado. Esto se realiza desde las características de la investigación y el proceso para culminarla, y se procuró que cada elemento de la metodología se encontrara relacionado. A continuación se detalla el paradigma: 


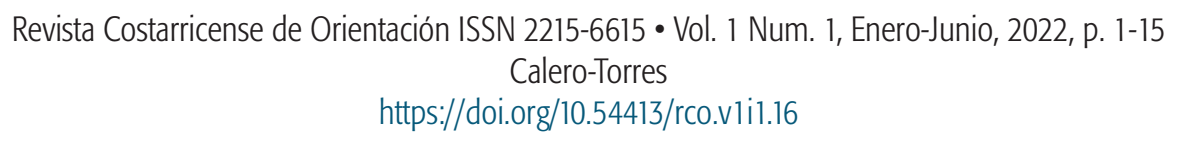

El paradigma naturalista posee un fundamento decididamente humanista para entender la realidad social de la posición idealista que resalta una concepción evolutiva y negociada del orden social. Percibe la vida social comola creatividad compartida de los individuos. El hecho de que sea compartida determina una realidad. (Ramírez, Arcila, Buriticá y Castrillón, 2004, p. 53)

En concordancia con el enfoque humanista que posee la Universidad Nacional de Costa Rica y los intereses del investigador, se utilizó este paradigma para brindar espacios de libre expresión a las estudiantes y así conocer su realidad en interacción con los otros factores; además están en armonía con la posición epistemológica deseada.

\section{Enfoque de investigación}

El enfoque utilizado responde al paradigma naturalista y a las necesidades de esta investigación, ya que permite conocer las realidades de las participantes desde sus propias historias; es por este motivo que se seleccionó el enfoque cualitativo, el cual, según Denzin y Lincoln (2012), es entendido como:

El término cualitativo implica un énfasis en las cualidades de los entes y en los procesos y significados que no pueden examinarse o medirse experimentalmente (si es que pueden medirse en absolutos) en función de cantidad, número, intensidad ofrecuencia. Los investigadores cualitativos subrayan la naturaleza socialmente construida de la realidad, las restricciones contextuales que dan forma a la investigación. (p. 62)

El nombre del enfoque se vincula con las cualidades, es decir, se refieren a los elementos naturales e innatos de la población y su relación con el fenómeno (la pregunta de la investigación). De esta forma se busca relacionar estos dos aspectos de manera directa: el reaccionar y el sentir de las personas ante una situación.

En este tipo de enfoque se encuentran las investigaciones que no se pueden o no se desean exponer ante situaciones de medición para llegar a las conclusiones del estudio; en vez de obtener numeraciones, se cuenta con etnografías.

Es por este motivo que algunos investigadores se inclinan hacia este enfoque, ya que desde sus criterios desean interactuar con la población (dependiendo del tipo de estudio por realizar). Denzin y Lincoln (2012), consideran que algunasformas de aplicarelenfoquees pormediode: "reutilizanlosenfoques, métodosytécnicasdelaetnometodología, la fenomenología, la hermenéutica, el feminismo, la rizomática, el deconstruccionismo, la etnografía, las entrevistas, el psicoanálisis, los estudios culturales, las encuestas, la observación participante, entre otros”. (p. 55)

La finalidad de estas técnicas o instrumentos es recolectar los datos desde las fuentes primarias con sus respectivas visiones de los acontecimientos, y con las interpretaciones que realicen a partir de su vivencia (cultura, ideología, nivel educativo, clase social, entre otros elementos que crean el marco de referencia de la persona, la construcción que realiza sobre el mundo y los distintos temas). Por este motivo, es que se recaba una gran cantidad de información (siempre y cuando sean aplicados correctamente) por medio de entrevistas, cuestionarios, grupos focales, estudios de casos y culturales o autobiografías, por nombrar algunos casos.

\section{Diseño de investigación}

En este capítulo se establece la metodología utilizada, la cual, como se ha recalcado, varía según la finalidad que posea el investigador sobre el trabajo. Al considerar el paradigma, el diseño y el enfoque estos se encuentran relacionados entre sí; además se toman en cuenta los propósitos establecidos, el adecuado planteamiento de la pregunta, los instrumentos por utilizar e incluso la elección del tema con los aspectos que lo integran. Cada uno de estos apartados permite tener una mayor claridad durante el proceso. 


\section{Revista Costarricense de Orientación ISSN 2215-6615 • Vol. 1 Num. 1, Enero-Junio, 2022, p. 1-15 \\ Calero-Torres \\ https://doi.org/10.54413/rco.v1i1.16}

Con respecto al diseño, Flick (2015) plantea que:

Un buen diseño tiene un foco claro y se construye en torno a una pregunta de investigación clara. El diseño y las preguntas permiten que la investigación reduzca el estudio a la cuestión esencial para responder a la pregunta. Un buen diseño hace que la investigación sea manejable en cuanto a los recursos y el tiempo y es claro en las decisiones sobre el muestreo y los porqués del uso de métodos particulares. (pp. 75-76)

De acuerdo con esta cita, cada una de las elecciones realizadas para la construcción del trabajo son de importancia, en este sentido, el diseño permite reducir la investigación en aspectos más concretos. Por lo tanto, esto contribuye a una mayor claridad, mantiene lo esencial y descarta la información innecesaria y evita una saturación de esta.

Asimismo, se indaga la teoría referente a los paradigmas naturalista y se elige por sus características el diseño narrativo. Según el fundamento de Hernández et al. (2014): "los diseños narrativos pretenden entender la sucesión de hechos, situaciones, fenómenos, procesos y eventos donde se involucra pensamientos, sentimientos, emociones e interacciones, a través de las vivencias contadas por quienes los experimentaron” (p. 487).

La intencionalidad principal de la investigación consiste en conocer la influencia vocacional que intervino en la elección de las estudiantes participantes. Por ende, al realizarse desde el enfoque cualitativo, surge un gran interés por los sentimientos y emociones que vivenciaron las estudiantes, las relaciones, las narrativas a nivel familiar y social, pero todo esto desde sus vivencias.

Dado a que se quiere estudiar el fenómeno relacionado con la elección de carrera, se realizó una serie de procedimientos, desde el paradigma naturalista, que permite conocer a las estudiantes como seres individuales que poseen un mundo interno e interactúan con el externo. Por ello, la fenomenología, como diseño de investigación, se considera el más adecuado para el alcance de los resultados; ya que faculta el acercamiento directo con la población en estudio y se adecua a los propósitos y al planteamiento de la pregunta. Además, permite trabajar de forma directa con las declaraciones de las estudiantes y no se modifica ninguna de estas ni se interpretan, son tomadas tal y como las expresan las participantes.

\section{Alcance de investigación}

Con respecto al alcance, se requiere la misma línea investigativa de los tres anteriores para desarrollar una congruencia lógica; es decir, desde el enfoque cualitativo y el paradigma naturalista. Por lo cual, tras una revisión de la literatura que se inició por el concepto de alcance, y además por la indagación de cada uno de los tipos que existe, se reconoce la importancia que posee. Como mencionan Hernández, Méndez, Mendoza y Cuevas (2017), "del alcance dependerá tu estrategia de investigación, incluido el diseño, los procedimientos y otros elementos” (p. 74). En consecuencia este alcance guía el trabajo que se realizó con la investigación, resalta aspectos de gran importancia como son los procedimientos, el accionar, y la fundamental metodología de la recolección de datos y los resultados de estos; por lo tanto, el investigador debe tener plena conciencia en la escogencia del alcance que desee.

Se selecciona el alcance explicativo, este es definido por Hernández et al. (2017) de la siguiente manera:

Los estudios explicativos son más que la descripción de conceptos o fenómenos o el establecimiento de relaciones entre variables; más bien, están diseñados para determinar las causas de los eventos y fenómenos físicos o sociales. Como su nombre lo indica, su interés se centra en explicar por qué ocurre un fenómeno y en qué condiciones se manifiesta, o por qué se relacionan dos o más variables. (p. 78) 
El alcance explicativo define una relación de causa-efecto en la cual se relaciona un fenómeno con el motivo de su origen. Esto, con relación a este trabajo, define la causa (fenómeno) de la selección vocacional de las estudiantes participantes y el factor o factores que propiciaron esta toma de decisión. Después de tener estas definiciones se incluye lo que sería la incidencia (el efecto, motivo por el cual ocurrió el fenómeno), lo que da como resultado la selección y la matrícula de la profesión.

Durante la investigación se abarcaron los tres factores vocacionales: personales, familiares y sociales; y se dará principal énfasis a los que predominen sobre los demás, la cual se considera la causa principal y da origen a una relación entre las categorías.

Aunque las fuentes primarias de información son las estudiantes, no se describió un perfil específico de cada una de ellas.

\section{Resultados y análisis}

A continuación se presenta el análisis de los resultados con la teoría. Es importante mencionar que se consideró la ética en el proceso indagatorio, por lo cual se utilizaron seudónimos en vez de los nombres reales, también se firmó por parte de las participantes un consentimiento informado y se les comentó que la información sería utilizada únicamente con fines de la investigación y no personales. Además, ante cualquier consulta podían contactar al investigador en cualquier momento de la investigación.

Aunquela entrevista no fuegrabada, la presencialidad le brinda al investigadorla oportunidad de observar el lenguaje no verbal, e identificar quizás gestos de emoción o disgusto. Al ser una entrevista semiestructurada, permite (si fuera considerado necesario) ampliar o indagar en alguna de las respuestas brindadas.

Finalmente, con las historias de vida se plasman algunas frases que se consideraron atinentes para ser analizadas dentro de la investigación.

\section{Tabla 2}

Información general de la población

Datos generales de la población en estudio

\begin{tabular}{lccccc}
\hline Edades & 17 años & 18 años & 19 años & & \\
Cantidad de estudiantes & 2 & 2 & 1 & & \\
Lugar de procedencia & Cartago & San Carlos & Sarapiquí & San Jerónimo & Heredia \\
Cantidad de estudiantes & 1 & 1 & 1 & 1 & 1 \\
\hline
\end{tabular}

Seguidamente se analizará cada uno de los factores de forma separada con su respectivo propósito, basados en las respuestas y en la teoría mencionada en la investigación. 


\section{Revista Costarricense de Orientación ISSN 2215-6615 • Vol. 1 Num. 1, Enero-Junio, 2022, p. 1-15 \\ Calero-Torres \\ https://doi.org/10.54413/rco.v1i1.16}

\section{Factor personal}

\section{El interés}

Se evidencia que el interés es primordial en la elección vocacional. Esto propició la inclinación hacia la carrera en estudio, y descartó otras opciones dentro de las ofertas académicas que brinda la Universidad Nacional. De modo metafórico, el interés se relaciona con la atracción hacia un “objeto”, en este caso sería esta ingeniería; y cada uno de sus elementos cautiva a las jóvenes como es el trabajo en el campo, la utilización del equipo e instrumentos de medición como el teodolito. Algunas frases etnográficas que respaldan lo anterior son: "Topografía siempre me llamó la atención, el trabajo que realiza me llama mucho la atención” (Sara, comunicación personal, 02 de marzo de 2020), "Durante el colegio cuando las universidades nos visitan hablaban de las carreras y entre todas esas, topografía me gustó mucho" (Emily, comunicación personal, 02 de marzo de 2020), las frases etnográficas citadas se obtuvieron en las historias vidas, como se logró apreciar en el apartado de referentes teórico de la presente investigación, el interés innato, nace de ellas propiamente y genera un nivel de expectativas y emociones.

\section{Autoconocimiento}

Es un elemento intrínseco. Este se entiende como la capacidad de la persona de reconocerse como ser humano; y reconocer sus fortalezas, oportunidades, debilidades e incluso amenazas. Tal, está estrechamente relacionada a la capacidad de las estudiantes para sobrellevar y culminar la profesión, considerando aspectos como la habilidad alfanumérica requerida en el plan de estudios, la fuerza física necesaria para el transporte de los instrumentos, el desarrollo de habilidades blandas como el trabajo en equipo, además de las habilidades “duras” para el trabajo en el campo por sí solo en días lluviosos o muy soleados, entre otros.

La introspección se considera oportuna para este autoanálisis que realiza cada persona o debería de ejecutar a la hora de tomar una decisión, comparando entre sí los tres factores y sus elementos; ya que el conjunto de los mismos son parte del proceso vocacional, rama de la disciplina de la orientación que se encarga del proceso de preparación a la incursión a la vida laboral.

$\mathrm{Al}$ consultarles a las entrevistadas sobre la permanencia en la carrera, las cinco manifestaron su deseo de finalizarla; sin embargo una de ellas mencionó que no era su primera opción en otro ítem de la entrevista. Pero, a pesar de esto, se evidencia un autoconocimiento en la joven, ya que poseía otro plan en su proyecto de vida. No obstante, tras un autoanálisis mencionado en la historia de vida, seleccionó la Ingeniería en Topografía, estando segura de su nueva decisión.

En cuanto al ítem dos, existen diversos factores externos que intervinieron en la decisión, como: las visitas a ferias vocacionales, las fantasías vocacionales desde la niñez, entre otros elementos externos que enriquece su bagaje de información. Así, las jóvenes recogen esta información y la incorporan a su marco interno de referencia; es decir, como ven este trabajo, que entienden de esta profesión, si aumentó o no el conocimiento.

Es de conocimiento empírico de toda persona profesional en Orientación que este proceso es continuo y constante; lo que permite la exploración, planificación de metas y construcción del yo interno que se encuentra en constante cambio. Las estudiantes requieren clarificar sus capacidades en el área de estudio, y la introspección se realiza para construir un panorama previo el cual les brinda un cierto nivel de seguridad y confianza a su vez, ya que salen de la zona de confort para enfrentar un nuevo reto del cual ya tienen una idea al menos de que esperar.

Por último, las estudiantes manifiestan, constantemente, encontrarse satisfechas de su elección en las historias de vida; $y$ mantienen expectativas del futuro a nivel personal y profesional, lo cual las mantiene motivadas y emocionadas. 


\section{Revista Costarricense de Orientación ISSN 2215-6615 • Vol. 1 Num. 1, Enero-Junio, 2022, p. 1-15 \\ Calero-Torres \\ https://doi.org/10.54413/rco.v1i1.16}

\section{Factor familiar}

Se considera la influencia familiar y comunicación de las estudiantes con estos miembros durante el proceso de ingreso a la carrera universitaria.

Para una vinculación con el apartado del marco metodológico, se indica la selección del paradigma naturalista como un elemento esencial en la estructura para la creación de la investigación (el mismo es reconocido y validado por el investigador); debido a que las participantes como seres humanas interactúan con sus familiares, se impregnan de la esencia, los valores inculcados y del currículum oculto que posee cada grupo. Por lo tanto, la influencia se realiza de forma directa o indirecta, y la comunicación es diaria en cualquiera de sus múltiples formas verbales o no verbales.

El análisis de la información obtenida se realizó con las historias de vida y el ítem 11 de la entrevista, en el cual respondieron ser apoyadas cuando fueron admitidas a la universidad, y que sus encargados las motivaron a seguir el proceso de admisión.

Existió un caso específico en el cual una joven comenta, al consultarle directamente si algún miembro influyó en su elección, que posee un primo arquitecto que es un ejemplo a seguir. Por lo tanto se evidencia la influencia en forma de admiración o inspiración.

Al finalizar el análisis entre los instrumentos y la teoría, se visualiza de forma concreta que la familia no fue un factor influyente absoluto en la elección de las estudiantes. Esta mención se respalda en los nueve ítems que tiene este factor en la entrevista, y en las narrativas de las historias de vida de cada una de ellas; no obstante, se recalca que la familia brindó apoyo, soporte, seguridad, independencia y la autonomía requerida, mas no insistieron para hacerlas cambiar de parecer o sus comentarios no fueron decisivos.

En cuanto a la categoría relacionada con la comunicación, las estudiantes comentaron que en determinados momentos, cuando conversaban con sus familiares, existieron sugerencias relacionadas con otras carreras. Cuatro estudiantes comentan, principalmente en la entrevista, que los familiares sugirieron otras profesiones, al inicio del proceso de admisión.

A la luz de la teoría del Instituto Nacional de Estadística e Informática de Perú (INEI, 2012) -que si bien no es un informe costarricense, se toma como un aporte de la realidad que viven las mujeres sin importar su país de procedencia-, se deriva que las estudiantes muestran predominancia en la selección de profesiones relacionadas con administración, salud, alimentación, entre otras.

Adicionalmente, las recomendaciones de sus familiares se dirigían a disciplinas con predominio femenino, inclusive ramas del Derecho, por su enfoque a velar por proteger el interés de alguien.

Sucede lo contrario en relación con la Arquitectura, área no tradicional para las mujeres desde la perspectiva del patriarcado, con los estigmas que poseen las personas según su sexo y lo esperado para estas personas, tal como se visualizó sobre el sexismo.

Además, existe una ruptura con el tradicionalismo, lo que evidencia -según las teorías- el surgimiento de los nuevos roles; por lo tanto, en los resultados de este elemento se evidencia que los familiares sí propusieron profesiones más tradicionales para el sexo de las estudiantes; sin embargo, al final ellas declinaron esas propuestas y su escogencia está relacionada con sus propios intereses y no con los de terceras personas.

\section{Factor social}

Se establece reconocer el conocimiento del medio y de los factores socioeconómicos como unidades de análisis, dado que poseen una estrecha relación entre sí. 


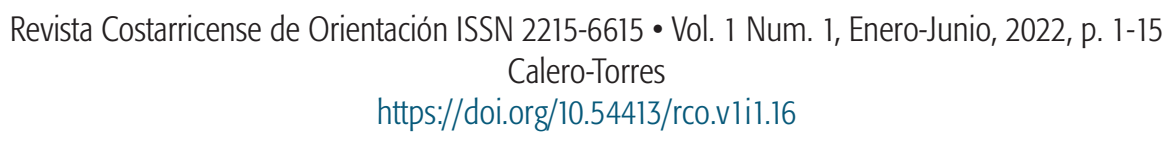

En la entrevista se les consultó por el conocimiento previo de la disciplina y surgieron respuestas como "ninguno" (Emily, comunicación personal, 26 de febrero de 2020), "normalmente veo programadas, directamente" (Joseline, comunicación personal, 26 de febrero de 2020), "sé que consistía en medir terrenos, se trabaja mucho en el campo" (Sara, comunicación personal, 26 de febrero de 2020), esta aparente carencia en la indagación previa se contrapone por el interés mostrado en la pertenencia de la carrera, el cual parece ser mayor la autodeterminación y el empoderamiento de las estudiantes.

Cuando un ser humano se propone una meta sucede un proceso intrínseco en el individuo, donde podría sentir emociones y sentimientos como miedo, incertidumbre, inseguridad o, por lo contrario, felicidad, ansiedad, entre otros. A la vez, cada persona es inigualable, holística en su esencia, y tiene su propia y única forma de ver el mundo, la cual influye en estas decisiones a corto, mediano y largo plazo. De esta manera, todos los seres humanos deben realizar un proceso vocacional adecuado, donde realicen las consultas que consideren necesarias a los profesionales encargados, sentir apoyo de parte de sus familiares y, además, estar seguros de sí mismos.

En relación con esta investigación, ingresar a una universidad puede ser sinónimo de superación u orgullo; y para culminar esta etapa, es necesario realizar un procedimiento que consiste en pagar el examen de admisión, la realización de este, la obtención de la nota de admisión, ingresar a la universidad y posteriormente a la carrera. Por consiguiente, al tomar en cuenta los tiempos de espera y los esfuerzos invertidos se determina que las jóvenes realizan una decisión planificada y no al azar.

Por lo tanto, esta influencia se asocia en mayor medida con autodeterminación impulsada por el interés de las jóvenes participantes en estudio.

\section{Conclusiones}

- Los factores vocacionales influyen en la elección de las estudiantes. Así, se determinó que refuerzan la determinación en las participantes, y que siempre se encuentran presentes de forma consciente o inconsciente al tomar una decisión.

- El factor personal presentó mayor influencia en las estudiantes. Durante el estudio las jóvenes abarcaron a plenitud las dos subcategorías y fueron enfáticas al mencionar el interés por la profesión; lo que evidencia el autoconocimiento constantemente al comentar debilidades en las áreas de matemáticas. Asimismo, se reforzaron sus gustos por el trabajo en el campo y por el nivel físico que requiere transportar los instrumentos de la disciplina.

- Por el contrario, el factor que mostró menor influencia en el proceso de carrera fue social. La mayoría de las participantes mencionaron no haber visto en su zona de procedencia demanda laboral, por lo cual no realizaron la decisión basada en un modelaje. Además comentaron ver escasa o nula la representación femenina, lo que apoya al argumento anterior. Asimismo, poseen ideas generales de las tareas de una ingeniera topográfica, incluso ya inmersa en el sistema educativo superior, lo que demuestra carencias en el conocimiento del medio dado que se encuentran en el primer nivel de la carrera.

- Las jóvenes son conscientes de que se encuentra en un mercado con predominancia masculina. Esto lo consideran un reto durante los estudios y la inserción en el mercado laboral, ya que son conscientes de que existe una serie de estereotipos para las mujeres en estos ámbitos.

- La elección vocacional fue un proceso planificado, puesto que las cinco participantes ya habían decidido entrar en esta profesión en la Universidad Nacional. 
- Las estudiantes, ante su empoderamiento, muestran no haber sido influenciadas por estereotipos sexistas que existen en la sociedad y de los cuales mostraron ser conscientes. Así, al ingresar a la profesión, rompieron los roles tradicionales asociados a su sexo, y satisficieron sus deseos y aspiraciones personales.

- En el área de orientación se concluye que es de suma importancia que las personas valoren todas las opciones (redes de apoyo, la creación de planes y la dedicación de tiempo para meditar al respecto) que poseen antes de tomar una decisión; puesto que una mala decisión podría conllevar consecuencias negativas como sentimientos de arrepentimiento, disgusto por las materias, atrasar sus proyectos de vida al tener que realizar el cambio de carrera, frustración, estrés, etc.

- La familia es un referente para el individuo, pero quien tomará la decisión final basada en sus propios deseos será la persona en la etapa de adultez joven; ya que goza de mayor autonomía para priorizar sus intereses.

- Las conclusiones y recomendaciones se consideran un logro en trascendencia, dado que este aporte didáctico permite ver una realidad que proviene directamente de la fuente primaria. De este modo, su valor teórico y relevancia social es irrefutable para futuras investigaciones.

\section{Recomendaciones}

- A los futuros profesionales de orientación, trabajar desde la igualdad de género y la ruptura de estereotipos. Se ha demostrado que la presión social puede ser determinante a la hora de tomar decisiones, por lo que las orientadas incursionarán en nuevos ámbitos (fuera de los tradicionales) si se les muestra que tienen la misma capacidad de sus compañeros.

- A las carreras de enseñanza, agregar en sus cursos temas relacionados con el fenómeno de las nuevas feminizaciones (los cambios de roles en las mujeres a través del tiempo) y de las carreras no tradicionales. Esto se considera oportuno para que las estudiantes universitarias amplíen sus conocimientos y no repliquen ideas tradicionales como las profesiones de cuido o enseñanza para el sexo femenino y las de fuerza o de campo para el sexo masculino.

- Ala Escuela de Topografía, Catastroy Geodesia, realizar campañas de inclusión y de sensibilización ante la presencia de mujeres en un campo con predominancia histórica masculina para evitar el machismo y el sexismo.

- A la Universidad Nacional, implementar campañas vocacionales o promocionales de la profesión dirigidas a las mujeres; ya que, ante los resultados se revela que la decisión es realizada por el interés de ellas mismas, por lo cual es pertinente captar su atención.

- A los y las profesionales de la orientación en los distintos campos laborales, realizar charlas o foros donde inviten ingenieras de las distintas áreas para que relaten su experiencia, lo cual serviría de inspiración.

- Darle continuidad a la muestra que participó, en trabajos futuros, para conocer su percepción de la carrera al poseer mayor conocimiento.

- Empoderar a las estudiantes de primer ingreso de la ingeniería, brindándoles herramientas para su permanencia.

- A los y las profesionales en orientación, reforzar los procesos de orientación vocacional en sus instituciones con información actualizada y no sexista.

- A la brevedad posible involucrar a la familia de los orientados en estos procesos para que sientan un apoyo desde el hogar. 


\section{Revista Costarricense de Orientación ISSN 2215-6615 • Vol. 1 Num. 1, Enero-Junio, 2022, p. 1-15 \\ Calero-Torres \\ https://doi.org/10.54413/rco.v1i1.16}

\section{Referencias}

Álvarez, M. (2017). Análisis del comportamiento de elección de carrera de los estudiantes de primer ciclo de Medicina de la Universidad Católica de Cuenca [Tesis de maestría, Universidad Católica de Santiago de Guayaquil]. Repositorio digital de la Universidad Católica de Santiago de Guayaquil. http://repositorio.ucsg.edu.ec/ bitstream/3317/8326/1/T-UCSG-POS-MGM-54.pdf

Cubillas, M., Valdez, E., Domínguez, S., Román, R., Hernández, A. y Zapata, J. (2016). Creencias sobre estereotipos de género de jóvenes universitarios del norte de México. Revista Diversitas. Perspectivas en Psicología, 12(2), 1-14. http://www.scielo.org.co/pdf/dpp/v12n2/v12n2a05.pdf

Denzin, N. y Lincoln, Y. (2012). El campo de la investigación cualitativa: Manual de investigación cualitativa. Gedisa

Flick, U. (2015). El diseño de investigación cualitativa. Ediciones Morata.

Fraile, A. y Vizcarra, M. (2009). La investigación naturalista e interpretativa desde la actividad física y el deporte. Revista de Psicodidáctica, 14(1), 119-132.http://www.redalyc.org/pdf/175/17512723008.pdf

Galilea, V. (s.f.). Orientación vocacional. http://www.crlnuevavida.es/crl/images/articulos pdf/Orientacion vocacional.pdf

Hernández, R., Fernández, C. y Baptista, P. (2014). Metodología de la investigación. Interamericana Editores.

Hernández, R., Méndez, S., Mendoza, C. y Cuevas, A. (2017). Fundamentos de investigación. Interamericana Editores

Instituto Nacional de Estadística e Informática. (2012). Profesiones o carreras universitarias. https://www.inei.gob.pe/ $\underline{\text { media/MenuRecursivo/publicaciones_digitales/Est/Lib1150/cap05.pdf }}$

Márquez, M. (2016). La inteligencia emocional: autoconocimiento, autoestima y empatia. https:// digibug.ugr.es/bitstream/handle/10481/45897/Miriam_L\%F3pez_M\%E1rquez. pdf;jsessionid=916AE70125F9DB76BE2C0DD424FF9BF9? sequence $=1$

Mejías, M. (2017). La evolución del estereotipo de la mujer en la historia de la publicidad [Trabajo fin de grado, Universidad de Valladolid]. Repositorio digital de la Universidad de Valladolid. https://uvadoc.uva.es/ bitstream/10324/24843/1/TFG-N.\%20747.pdf

Ramírez, L., Arcila, A., Buriticá, L. y Castrillón, J. (2004). Paradigmas y modelos de investigación. Guía didáctica y módulo (2.a $\quad$ ed.). $\quad \underline{\text { http://virtual.funlam.edu.co/repositorio/sites/default/files/ }}$ repositorioarchivos/2011/02/0008paradigmasymodelos.771.pdf

Reyes, I. y Novoa, A. (2014). Orientación vocacional. Editorial de la Universidad Central.

Schuster, A., Puente, M., Andradra O. y Maiza, M. (2013). La metodología cualitativa, herramienta para investigar los fenómenos que ocurren en el aula. La investigación educativa. Revista Electrónica Iberoamericana de Educación en Ciencias y Tecnologia, 4(2), 109-139. http://www.exactas.unca.edu.ar/riecyt/VOL\%204\%20 NUM\%202/TEXTO\%207.pdf

Tustón, M. (2016). La comunicación familiar y el asertividad de los adolescentes de noveno y décimo año de educación básica del Instituto Tecnológico Agropecuario Benjamin Araujo del cantón Patate [Tesis de grado, Universidad Técnica de Ambato]. Repositorio digital de la Universidad Técnica de Ambato. http://repositorio.uta.edu. ec/bitstream/123456789/20698/2/COMUNICACION\%20FAMILIAR\%20Y\%20ASERTIVIDAD\%20 -\%20copia.pdf 